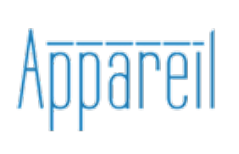

Appareil

$14 \mid 2014$

Esthétiques latino-américaines : penser à rebours

\title{
L'innervation technique du regard et de la mémoire en Amérique latine
}

Natalia Calderón

\section{(2) OpenEdition}

1 Journals

Édition électronique

URL : http://journals.openedition.org/appareil/2098

DOI : 10.4000/appareil.2098

ISSN : 2101-0714

Éditeur

MSH Paris Nord

Référence électronique

Natalia Calderón, «L'innervation technique du regard et de la mémoire en Amérique latine », Appareil [En ligne], 14 | 2014, mis en ligne le 10 décembre 2014, consulté le 30 juillet 2020. URL : http:// journals.openedition.org/appareil/2098; DOI : https://doi.org/10.4000/appareil.2098

Ce document a été généré automatiquement le 30 juillet 2020.

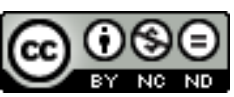

Appareil est mis à disposition selon les termes de la Licence Creative Commons Attribution - Pas d'Utilisation Commerciale - Pas de Modification 4.0 International. 


\title{
L'innervation technique du regard et de la mémoire en Amérique latine
}

\author{
Natalia Calderón
}

« La photographie moderne a non seulement

élargi notre

champ de vision, mais, ce faisant, elle l'a adapté à la situation de l'homme à l'âge de la technique »

Siegfried Kracauer ${ }^{1}$

1 Le rapport qu'on prétend développer entre l'Amérique latine et l'appareil photographie prend pour départ une hypothèse, à savoir qu'il existe une contemporanéité entre la construction des premiers imaginaires nationaux latino-américains et l'arrivée de l'appareil photographique. Si tel est le cas, les conséquences de cette contemporanéité sont un enjeu majeur qu'on essayera de déployer.

2 C'est au milieu du XIX siècle que les nations latino-américaines commencent à se structurer après leur période indépendantiste. Pour cela a été nécessaire la création d'imaginaires nationaux, la délimitation des frontières et l'idée aussi de se mettre «à l'ordre du jour ", c'est-à-dire de mettre en place les idéaux modernes qui venaient d'Europe. C'est dans ce contexte que l'appareil photographique arrive en Amérique latine, et quand on dit " arrive » c'est parce qu'il vient d'Europe en tant que symbole de la modernité européenne. C'est à cause de cela que les gouvernements nationaux se sont appropriés rapidement ce nouvel appareil. Les conséquences de cette appropriation n'ont pas simplement été de l'ordre de l'outil qui sert a exemplifier des problématiques préalables, mais plutôt, et cela est notre thèse, qu'il s'agit d'un appareil qui a été capable d'innerver la conduite et la façon de penser de l'homme latinoaméricain. C'est dans ce sens qu'on proposera une idée d'appareil photographique qui critiquera la conception générale qu'on a de ce dernier en tant qu'outil. 


\section{L'appareil photographique : outil ou appareil qu'innerve le regard?}

3 Les concepts d'outil et de prothèse sont couramment utilisés pour parler des propriétés de l'appareil photographique. C'est ainsi que Baudelaire signale dans son célèbre passage du Salon de 1859 sur la photographie:

Qu'elle enrichisse rapidement l'album du voyageur et rende à ses yeux la précision qui manquerait à sa mémoire, qu'elle orne la bibliothèque du naturaliste, exagère les animaux microscopiques, fortifie même des quelques renseignements les hypothèses de l'astronome; qu'elle soit enfin le secrétaire et le garde-note de quiconque a besoin dans sa profession d'une absolue exactitude matérielle, jusquelà rien de mieux. Qu'elle sauve de l'oubli les ruines pendantes, les livres, les estampes et les manuscrits que le temps dérobe, les choses précieuses dont la forme va disparaître et qui demandent une place dans les archives de notre mémoire, elle sera remerciée et applaudie. ${ }^{2}$

Ce passage nous montre comment l'appareil photographique est présenté comme un auxiliaire des sciences, comme un garde-note qui vient remplir les déficiences de la mémoire, une archive parmi d'autres en définitive. Mais l'appareil photographique est bien plus que cela, car si l'on considère son fonctionnement, on peut se rendre compte qu'il n'est pas simplement un supplément ou prolongement de capacités humaines comme une loupe ou un marteau. Au contraire, cet appareil vient à dépasser les capacités humaines, en présentant des aspects insoupçonnés du réel, et en même temps on peut dire que la photographie elle-même donnera forme au réel, c'est-à-dire que la photographie sera productrice du réel.

5 Si le fondement de son utilisation par les gouvernements nationaux en Amérique latine a bien été cet aspect prothétique et archivistique, il y a néanmoins un autre côté encore, inattendu, qu'il faut développer pour mieux comprendre la problématique de la photographie en Amérique latine. Car la question de l'utilisation de la photographie ne permet en effet pas de comprendre complètement sa spécificité en tant qu'appareil qui produit des images techniques qui sont différentes de tout autre type d'images. Si l'on se concentre sur cet aspect technique de la photographie, on pourra démêler sa vraie présence, c'est-à-dire son rôle à l'intérieur de la nouvelle sensibilité qui naît avec la photographie. Ce rôle est fondamental pour pouvoir comprendre comment la photographie s'installe en Amérique latine et comment elle a été capable de donner forme à une nouvelle sensibilité. Cette sensibilité en étant contemporaine de la formation de premières nations américaines va accompagner tout le développement de ces nations. Voilà pourquoi il est important d'établir le fonctionnement de la photographie.

6 La problématique centrale est alors : la photographie est un outil ? une prothèse ? ou un appareil d'innervation humaine? Le fil conducteur qui nous permettra d'essayer de répondre à cette question sera celui de l'externalisation : nous essayerons de savoir si la photographie met vraiment en œuvre une procédure d'externalisation soit de la mémoire soit de la vue. 


\section{Le concept d'outil}

7 Les concepts d'outil et de prothèse sont définis comme une externalisation des capacités humaines, car cette mise à part rend possible une meilleur performance de ces capacités. Dans ce sens, la photographie a été largement considérée comme une externalisation de la mémoire collective, son fondement : la photographie en tant que reproduction exacte du réel permet la création des archives, c'est-à-dire des mémoires collectives externes dans un support matériel. Cette possibilité de mise en mémoire est considérée comme infinie, contrairement à la mémoire humaine qui présente des limites et qui en plus présente l'inconvénient d'être déformée par les interprétations subjectives. Au contraire, l'archive photographique est une mémoire externe à l'homme et en conséquence elle est loin des interprétations subjectives car il s'agit d'une reproduction objective de type mécanique.

D'autre part, l'appareil photographique peut être considéré comme un outil qui en prolongeant l'œil va au-delà des possibilités de la vision naturelle.

9 On a donc deux modes d'externalisation des capacités humaines à partir de l'appareil photographique, à savoir en tant qu'outil ou prothèse de la mémoire et en tant qu'outil ou prothèse de l'œil et de la vue, dans le deux cas l'appareil vient remédier une déficience humaine.

Leroi-Gourhan, théoricien de l'outil, va définir cette extériorisation ainsi :

Toute l'évolution humaine concourt à placer en dehors de l'homme ce qui, dans le reste du monde animal, répond à l'adaptation spécifique. Le fait matériel le plus frappant est certainement la «libération» de l'outil, mais en réalité le fait fondamental est la libération du verbe de cette propriété unique que l'homme possède de placer sa mémoire en dehors de lui-même, dans l'organisme social. ${ }^{3}$

Leroi-Gourhan fait une distinction entre outil et mémoire, il ne conçoit pas l'outil comme un type de mémoire. Pour lui la mémoire se trouve dans l'homme en tant qu'inscription d'un programme. Il s'agit dans ce cas de la mémoire collective et individuelle qui détermine le comportement de l'espèce. Le langage dans ce sens est une extériorisation du programme qui permet une libération par rapport au vécu. Par contre, l'outil permet une libération au niveau génétique de l'homme, l'outil va libérer l'homme de contraintes zoologiques qui le lient à l'espèce. Donc, mémoire externe rendue possible par le langage et outil sont deux types de libération de l'homme par rapport à l'espèce. La première agit au niveau du programme, le deuxième au niveau biologique.

Le premier pas de cette libération est le fait de placer sa propre mémoire en dehors de soi-même dans le monde social; c'est un premier pas qui libère l'homme de la génétique. Avec les nouvelles technologies arrive un nouveau stade où: "la main déclenche un processus programmé dans les machines automatiques qui non seulement extériorisent l'outil, le geste et la motricité, mais empiètent sur la mémoire et le comportement machinal $»^{4}$. À cet égard, on peut penser à une extériorisation de toutes les capacités humaines et qui implique même le comportement.

13 Suivant Leroi-Gourhan, on peut donc comprendre l'appareil photographique comme une machine automatique qui est capable d'extérioriser l'outil en même temps que la mémoire et le programme. Mais ce serait oublier un aspect fondamental du fonctionnement de l'appareil, à savoir « l'innervation du corps ». 
Certes la photographie met en place une extériorisation de la perception et de la mémoire visuelle et dans ce sens on est d'accord avec Leroi-Gourhan. Notre désaccord vient plutôt de la réduction de la problématique à l'extériorisation sans tenir en compte d'un facteur très important, à savoir une procédure de réversibilité de cette mise en mémoire. L'outil externalise des capacités humaines en même temps qu'il introjecte les nouvelles possibilités que l'outil déploie. Car l'outil va créer des nouvelles formes de construction du réel qui échappent à ses capacités originelles. Telle est la thèse de Jean-Louis Déotte et sa critique ${ }^{5} \mathrm{du}$ concept d'outil chez Leroi-Gourhan.

Leroi-Gourhan signale pourtant :

Conçue généralement comme un phénomène historique, de signification technique, l'apparition du chariot, de la charrue, du moulin, du navire est aussi à considérer comme un phénomène biologique, une mutation de cet organisme externe qui se substitue chez l'homme au corps physiologique. ${ }^{6}$

Cette "mutation» nous fait penser que pour lui l'externalisation effectue une modification biologique de l'individu, modification qu'on peut penser comme une introjection de l'outil qui modifie l'aspect biologique de l'individu. Dans ce sens, la technique modifie la structure biologique de l'homme. Dans le cas de la photographie, cette modification est de l'ordre de la perception, car la photographie modifie la façon de percevoir le monde. Mais, le problème c'est que Leroi-Gourhan pense toujours depuis le point de vue des capacités humaines, il en va de même chez Baudelaire pour qui la photographie est une aide, une servante, un auxiliaire, l'inouï étant réservé aux domaines de l'imagination créatrice, seule capable de produire des nouveaux mondes. Cependant l'appareil photographique est bel et bien capable de créer de nouvelles possibilités inouïes pour l'homme.

Une critique de cette idée d'outil ou de prothèse peut être mise en place depuis le point de vue de la théorie de l'innervation chez Benjamin et depuis le point de vue de la mémoire involontaire chez Proust, car tous les deux mettent en place cet aspect inouï que possède l'appareil photographique, aspect qui a été capable de modifier la perception au XIX ${ }^{e}$ siècle.

\section{Walter Benjamin et l'innervation technique}

18 Le concept d'innervation que Benjamin s'approprie pour construire une théorie capable d'expliquer les changements perceptifs qui ont eu lieu dans la modernité est un concept qui a son origine dans les traités de physiologie du XIX siècle.

9 Le dictionnaire de l'Académie royale espagnole va définir le concept d'innervation de la manière suivante :

Innerver :

tr. physiol. L'action d'un nerf sur une zone d'un organisme animal.

Innervation :

Biol. L'action du système nerveux dans les fonctions des autres organes du corps animal.

Il s'agit alors d'un concept qui explique le fonctionnement du système nerveux à partir des nerfs qui transmettent les impressions des sens. J.-P. Morat dans son Traité de physiologie. Fonctions d'innervation, signale : « C'est lui [le système nerveux] qui décide du moment où l'énergie accumulée par l'être vivant doit se libérer, c'est-à-dire quitter la 
substance et réaliser ses effets moteur. Il en décide d'après les informations à lui communiquées par les organes des sens $[\ldots] »^{7}$.

On peut en conclure que l'innervation est un moyen de transmission des informations qu'apportent les sens, les sens «absorbent au dehors » et transmettent au nerf cette information qui finalement arrive au cerveau. Ce qui est important dans cette idée, c'est que le cerveau génère une réponse motrice. Par exemple, la perception visuelle ne doit pas seulement être interprétée comme une pure visualité mais plutôt comme comportant un double aspect visuel et moteur. Morat définit les phases de ce cycle :

Il implique successivement un phénomène extérieur d'impression, un phénomène intérieur de sensation, un nouveau phénomène extérieur de réponse motrice à l'impression, suivi lui-même d'un nouveau phénomène intérieur de sensation qui enregistre le mouvement accompli. ${ }^{8}$

De manière parallèle, ce concept est aussi utilisé par Freud comme le signalent Laplanche et Pontalis dans leur Vocabulaire de la Psychanalyse: "Innervation: Terme utilisé par Freud dans ses premiers travaux pour désigner le fait qu'une certaine énergie est véhiculée vers telle ou telle partie du corps, y produisant des phénomènes moteurs ou sensitifs $»^{9}$

Or, il est intéressant que Benjamin signale qu'il doit au théoricien de l'art allemand Konrad Fiedler son concept d'innervation. Ainsi dans son Programme pour un théâtre d' enfants prolétarien :

Konrad Fiedler a montré le premier, dans ses Écrits sur l'art, que le peintre n'est pas un individu qui aurait une vision plus naturaliste, plus poétique ou plus extatique que des autres, mais plutôt un homme sachant regarder de plus près avec la main quand l'œil se paralyse, et qui transfère l'innervation réceptive des muscles de la vue dans l'innervation créatrice de la main. ${ }^{10}$

Bien que le concept d'innervation soit attribué à Fiedler, il est nécessaire de savoir que Fiedler n'utilise pas explicitement le mot « innervation » dans ses Essais sur l'art ${ }^{11}$, donc on peut conclure que Benjamin fait une synthèse des idées fondamentales que Fiedler développe dans son livre, en mettant en rapport ces idées avec le concept d'innervation qui vient de la physiologie.

En conséquence, qu'est-ce qui amène Benjamin à interpréter l'œuvre de Fiedler en termes d'innervation? D'abord, il est nécessaire de comprendre que le contexte du livre de Fiedler est la création artistique. La création artistique est chez Fiedler une procédure dans laquelle la visibilité est matérialisée en une activité, à savoir dans la production d'un objet artistique. L'artiste est celui qui est capable non pas seulement de « voir » et de penser le monde depuis un point de vue intellectuel, mais surtout celui qui peut conduire le visuel au champ de la motricité en produisant ainsi une œuvre. Fiedler signale :

Il n'y a que par l'activité que la représentation d'un objet dans lequel la visibilité apparaît s'efface complètement et que la visibilité accède à une forme autonome de l'être. L'artiste en fait l'expérience sur lui-même. Plus il se sent impliqué - non plus uniquement par l'œil, ou l'imagination, mais par sa personne tout entière, par les possibilités de sentir de tout son corps, par l'activité de ses mains - dans un processus qui va de la perception visuelle à la présentation visible extérieurement, plus il s'éloigne de toutes les relations avec les choses qui exerçaient auparavant une emprise sur lui. Sa participation à la visibilité des choses doit dépasser la simple perception, la simple représentation, elle doit le rendre actif, d'une activité extériorisée. ${ }^{12}$ 

une sorte de rétroalimentation, car les appareils techniques introduisent une nouvelle perception qui s'étend à tous les ordres de l'humain et de l'inhumain (car la technique oblige l'homme à suivre son mouvement machinal) en même temps que l'homme doit générer des appareils qui lui permettent de s'introduire dans ce nouvel aspect de l'époque.

31 C'est ainsi que pour Benjamin le cinéma est le moyen dont l'homme se sert pour pouvoir accéder à cette nouvelle époque de la reproductibilité technique qui est la modernité :

Une fois de plus, l'art est au service de cet apprentissage. Et notamment le cinéma. Sa fonction est de soumettre l'homme à un entraînement; il s'agit de lui apprendre les aperceptions et les réactions que requiert l'usage d'un appareillage dont le rôle s'accroit presque tous les jours. Faire de l'immense appareillage technique de notre époque l'objet de l'innervation humaine, telle est la tâche historique au service de laquelle le cinéma trouve son sens véritable. ${ }^{13}$

En définitive ce que Benjamin nous dit, c'est que la modernité se caractérise pour un nouveau mode de perception déterminé par la technique (seconde technique) qui introduit des nouvelles vitesses, entièrement caractérisées selon Benjamin par l'idée de 
choc, et telles qu'elles sont décrites dans ses textes sur Baudelaire où le poète se plonge dans la foule et subi une sorte de choc électrique.

Or, c'est le cinéma qui permettra à l'homme de s'entraîner au nouveau mode de vie, car le cinéma possède une structure de choc, les images s'enchaînent et se succèdent à grande vitesse, l'œil n'arrive pas à percevoir une image quand celle-ci a déjà disparu et que s'enchaîne une nouvelle.

En définitive, le cinéma a comme rôle fondamental de réussir à innerver l'homme avec les appareils techniques. Si comme on l'a déjà signalé, le concept d'innervation dans son aspect physiologique renvoie à l'action des nerfs sur les organes du corps, Benjamin de la même façon pense que le cinéma acquiert la même fonctionnalité qu'un nerf quand il transmet un mouvement moteur qui part d'une visualité pour finir dans un geste. De cette manière on peut considérer le caméraman comme un producteur des gestes que vont innerver le spectateur, gestes qui pénètrent le réel. De la même façon que le chirurgien pénètre les tissus, le caméraman saisit l'inconscient visuel et produit des images et gestes insoupçonnés.

Telle est l'innervation chez Benjamin, car le cinéma nous montre des images techniques discontinues, en perpétuel mouvement, ces images traduisent le mouvement de la vie moderne, images perçues par le spectateur du cinéma et qu'innerve la perception de ce dernier générant ainsi un geste qui lui permettra de s'introduire dans la vie moderne. Benjamin signale dans «L'œuvre d'art à l'ère de sa reproductibilité technique » :

Car c'est devant un appareil que la grande majorité des citadins doit, dans les bureaux comme dans les usines, abdiquer son humanité pendant la durée de sa journée de travail. Le soir, ce sont ces mêmes masses qui remplissent les salles de cinéma. ${ }^{14}$

La vie moderne et le travail introduisent en l'homme un automatisme qui lui est propre, machinal ; l'homme doit s'adapter en laissant de cette façon une partie de son humanité, en générant une sorte de symbiose entre l'homme et la machine. Benjamin signale : «Parmi les fonctions sociales du cinéma, la plus importante consiste à établir un équilibre entre l'homme et les appareils $»^{15}$.

Dans ce contexte, Charlie Chaplin est pour Benjamin un modèle d'innervation entre l'homme et la machine: lorsque dans son film Les temps modernes on observe Chaplin suivre avec difficulté le rythme accéléré des machines, on voit les difficultés du récent procès d'innervation au début $\mathrm{du} \mathrm{xx}^{\mathrm{e}}$ siècle.

Benjamin signale en ce sens :

Sa signification exceptionnelle réside en ceci que l'ensemble des gestes de l'homme - envisagé dans son comportement corporel autant que spirituel - est " assemblé » mécaniquement dans la structure du film. Voilà ce qui fait la nouveauté du gestus de Chaplin: il décompose le mouvement expressif de l'homme en une suite d'innervations infimes. Chacun de ses mouvements singuliers se compose d'une suite, en saccade, des mouvements fractionnés. ${ }^{16}$

De cette façon Chaplin est innervé par la machine car, quand ce dernier finit son travail, ses mouvements corporels finalement incorporent le rythme et le mouvement de la machine. Tel est l'aspect gestuel du concept d'innervation chez Benjamin, car le cinéma ne génère pas simplement un nouveau type de perception mais génère aussi des images capables d'innerver les gestes humains. Et voici le moment politique du concept d'innervation que Benjamin appellera «révolutionnaire »: «Les révolutions sont les 
innervations de l'élément collectif ou, plus exactement, les tentatives d'innervation de la collectivité qui pour la première fois trouve ses organes dans la seconde technique " ${ }^{17}$. C'est-à-dire l'innervation comme révolution ou la révolution comme innervation homme-technique, sorte d'équilibre entre l'homme et les forces de la nature rendu possible par l'innervation.

On peut dire que telle est l'utopie technique chez Benjamin, à savoir trouver l'harmonie entre l'homme et son milieu à partir de la technique.

Alors, est-ce qu'on peut finalement adopter le concept d'innervation pour parler de l'appareil photographique?

La théorie de la photographie chez Benjamin présente deux aspects, et le premier est un aspect qui prend justement en considération l'idée d'entraînement, ou plus précisément l'idée de la photographie en tant que barrière protectrice en face des sensations qui viennent de l'extérieur. Car le concept d'innervation chez Benjamin suppose une sorte de symétrie entre la vie moderne pleine de stimuli ou chocs et le cinéma qui nous prépare à faire face à ces stimuli. Il en va de même avec la photographie qui est pour Benjamin une sorte de choc posthume. Cette théorie de la photographie chez Benjamin a comme fondement la théorie freudienne de la conscience et des traces mnésiques. Elle est développée principalement dans ses textes sur Baudelaire.

L'intérêt de Benjamin pour Freud vient du fait que ce dernier développe, surtout dans son texte $A u$ delà du principe du plaisir, l'idée que conscience et trace mnésique sont contradictoires.

On peut dire que le modèle théorique de l'appareil photographique est freudien, car il nous présente un double aspect de la photographie : la conscience, d'une part, et la trace mnésique inconsciente, d'autre part.

\section{La photographie-conscience}

Chez Freud la conscience a une localisation dans la couche la plus extérieure du système nerveux, sa tâche est de protéger l'organisme des stimuli extérieurs : " pour l'organisme vivant, parer aux stimuli est une tâche presque plus importante que celle de recevoir les stimuli $\aleph^{18}$. L'excès des stimuli met l'organisme en danger, le rôle de cette couche est d'amortir les stimuli pour de cette façon maintenir une sorte d'équilibre. Cette idée de conscience en tant que couche protectrice est reprise par Benjamin pour développer son idée de photographie-conscience. Mais cette idée il faut la considérer depuis le point de vue des changements sociaux du XIX ${ }^{e}$ siècle comme la naissance de la vie moderne, les grandes villes, la foule et bien sûr la reproductibilité technique. C'est la crise de l'expérience en définitive où la reproductibilité technique et donc la photographie ont un rôle majeur. La reproductibilité va générer une absence de traces, car chez Freud «le fait de devenir conscient et celui de laisser une trace mémorielle sont, pour un même système inconciliables. Ainsi pourrions-nous dire que dans le système Cs le processus d'excitation devient conscient, mais ne laisse aucune trace permanente ${ }^{19}$. D'après Benjamin, la modernité est un état de conscience, où il n'y a plus de traces, et donc pas d'expérience véritable. L'expérience véritable est celle $\mathrm{du}$ récit, ancien mode de réception du phénomène où celui qui raconte des histoires laissait en même temps une trace. Cette expérience véritable construisait une tradition 
et donc une mémoire collective ancrée dans les couches les plus profondes de l'homme. C'est un type de mémoire où l'expérience particulière se fond dans l'expérience collective.

Par contre, dans l'expérience vécue de la vie moderne l'homme doit affronter la multiplicité de stimuli en se protégeant, et tel est justement l'intérêt de la photographie qui vient même empêcher l'expérience véritable. Benjamin introduit le concept d'information pour parler de cette espèce de blocage de l'expérience. L'information essaye de transmettre l'événement à l'état " pur » de sorte que le lecteur de journaux ne peut pas incorporer cette information à sa propre expérience. C'est ce mécanisme que la photographie met en place en nous présentant des événements décontextualisés. C'est un mécanisme du choc inaugurant un nouveau type de perception que Benjamin appellera "perception traumatisante $»^{20}$ car, de la même façon que la vie moderne pleine de stimuli exige un état constant de conscience pour pouvoir réagir de façon adéquate, traverser une rue suppose une attention au trafic, au signalétiques, au passants etc. C'est ainsi que la photographie, avec un seul déclic, capture un instant, une découpe de l'événement. Cette idée est développée par Benjamin dans son texte Sur quelques thèmes baudelairiens où il revient à l'idée d'innervation. La ville moderne et ses stimuli ont besoin d'une réponse motrice, cette réponse motrice est caractérisée par Benjamin par le "déclic instantané du photographe $»^{21}$ qui "confère à l'instant une sorte de choc posthume ${ }^{22}$. Ce mouvement automatique du déclic sera pour Benjamin une réponse motrice aux stimuli et en même temps un entraînement aux réactions motrices nécessaires pour vivre dans la ville moderne.

On a donc une photographie de la conscience qui, d'une part, protège l'homme de la menace des stimuli en amortissant l'événement, et qui, d'autre part, a un rôle d'entraînement moteur en face des stimuli.

Le déclic est alors le modèle d'innervation photographique car c'est une réponse motrice qui inaugure une nouvelle sensibilité. Car le déclic est un geste qui signale un mode de perception, c'est l'introjection de l'appareil photographique qui va créer une perception faite à partir des prises de vue discontinues. On verra plus tard comment Proust reconstruit sa mémoire à partir des différentes prises de vue ou des clichés comme il les appelle.

49 Cette idée est donc très importante pour comprendre l'ancrage du modèle photographique en Amérique latine, où la photographie est contemporaine de la construction des nations américaines. Ces nations, si l'on parle en termes benjaminiens, sont nées avec une crise de l'expérience, avec une rupture avec la tradition indienne et même avec la tradition coloniale, avec donc comme résultat une perception innervée par l'appareil photographique. Si l'on prête attention aux photographies de l'époque, on se rend compte qu'il s'agit toujours de prises de vue qui essayent de montrer la modernité des nations en oubliant en même temps toute relation avec le passé. Les autres types de photographies qui montrent les peuples indigènes ont toujours un regard étranger qui essaye de montrer la séparation entre le photographe, l'appareil (symbole de la modernité) et le sujet photographié (l'Indien) qui appartient à une autre époque, à une autre sensibilité. On peut penser au déclic du photographe comme à un geste moteur qui essaye de s'habituer à cette nouvelle vie qui naît avec l'idée de nation et qui essaye de se mettre à jour, c'est-à-dire de se conduire de manière suffisamment moderne. 


\section{La photographie comme inconscient optique et mémoire involontaire}

50 On vient de signaler que chez Benjamin il y a deux photographies, la première est celle de la conscience comme la poésie de Baudelaire, la deuxième est une photographie qui n'a pas un rôle de protection et qui n'est pas non plus une sorte d'entraînement à la vie moderne, il s'agit plutôt d'un type de photographie qui mettrait en place justement ce que la photographie-conscience va essayer d'empêcher, c'est-à-dire qu'il s'agit d'une photographie de l'expérience authentique.

51 Benjamin prend deux exemples clés pour parler de ce type de photographie, à savoir la construction de sa propre mémoire et le concept de mémoire involontaire chez Marcel Proust, auxquels il faut ajouter son concept d'inconscient optique qui nous va rapprocher de l'idée d'objet chez Proust.

On vient de parler d'une photographie-conscience en tant que mode d'innervation. Que peut-on dire de la photographie en tant qu'inconscient optique chez Benjamin?

La thèse centrale que Benjamin met en place à partir de la photographie est une critique du concept de sujet conscient. La mémoire avait toujours été pensée à partir de la médiation de l'homme comme centre et noyau de sa construction; or la photographie met en question ce noyau subjectif en l'extériorisant.

54 La photographie est une prise de vue automatique et externe, tandis que l'innervation implique une introjection de l'appareil photographique et donc de ce processus perceptif de prise de vue automatique. Benjamin comme Proust introjecte l'appareil et c'est ainsi que sa perception et sa propre construction de la mémoire sont déterminées par l'appareil à un point tel que les événements les plus importants d'une vie sont ceux qui ont laissé une trace, une «prise de vue » qui n’a pas été prise par le sujet lui-même mais plutôt par autrui.

Pour pouvoir comprendre cette idée, il faut reprendre la théorie freudienne de la conscience car cette "prise de vue» de notre vie est prise par autrui parce que c'est une trace et que toute trace s'oppose à la conscience. C'est cet aspect inconscient, ou cette « mémoire involontaire » comme l'appelle Proust, qui prend en charge cette prise de vue automatique. Le sujet n'a pas conscience de cette prise qui pourtant laisse une trace.

À cet égard, on peut dire que Benjamin et Proust ont un appareil perceptif de type photographique. Innervés par l'appareil, ils construisent leur mémoire à partir de prises de vues automatiques qu'il faut développer après-coup. Le développement de ces prises de vue est, chez Benjamin, le nœud de ses thèses sur l'histoire. Il en va de même avec Proust dont la Recherche n'est qu'un développement des prises de vue de sa vie. C'est ainsi que Brassaï signale à propos de Proust :

À la lumière de la photographie un nouveau Proust m'est apparu, une sorte de photographe mental, considérant son propre corps comme une plaque ultrasensible, qui sut capter et emmagasiner dans sa jeunesse des milliers d'impressions et qui, parti à la recherche du temps perdu, consacra tout son temps à les développer et à les fixer, rendant ainsi visible l'image latente de toute sa vie, dans cette photographie gigantesque que constitue À la recherche $d u$ temps perdu ${ }^{23}$. 

discontinuité, car c'est un morceau isolé du réel sans rapport aux autres morceaux. De la même manière, Benjamin considère que ses textes sur son enfance à Berlin ne constituent pas une biographie, car une biographie a nécessairement une continuité et une fluidité dans le temps. La photographie arrache l'objet photographié à son contexte et le rend ubiquitaire. De la même façon, ces prises de vue qui construisent la mémoire sont arrachées à la fluidité du temps, elles sont des images découpées du contexte où elles sont nées et rangées dans cette mémoire des traces.

concept d'automatisme lié à l'idée de photographie est très important car, comme on vient de le voir chez Benjamin, le déclic de la photographie instantanée, comme l'allumette qui prend feu avec un seul geste, sont une face de la perception et de l'innervation en général qui détermine la nouvelle sensibilité de la modernité. Le geste moteur qui correspond au déclic est introjecté et c'est la mémoire involontaire qui met en place ce mouvement automatique des prises de vue. On peut dire alors que le déclic est le modèle perceptif au XIX ${ }^{e}$ siècle.

Une autre conséquence majeure de cette introjection de l'appareil photographie dans la perception du xIX ${ }^{e}$ siècle est l'objectivité de l'appareil, car il n'y a pas d'intervention humaine, c'est la machine qui prend des images qui reproduisent le réel. On ne s'arrêtera pas ici sur la question de savoir si l'appareil est vraiment objectif ou pas, le point étant qu'on ne peut pas négliger le fait qu'il s'agit d'images-techniques. C'est cette objectivité introjectée dans le mode de perception qui permet d'avoir des clichés de sa propre vie prises par autrui, le sujet n'intervenant pas dans la prise de vue. Il s'agit d'une objectivité qui n'a pas nécessairement de rapport avec la reproduction exacte du réel, mais plutôt avec une mise à distance du sujet conscient à cause d'un fonctionnement automatique. Cette conception du sujet qui perçoit est plus proche de celle d'un automate que d'un sujet-pensant.

Benjamin va interpréter cette objectivité comme un "être-en-dehors-de-nous ", comme une sorte de prise de distance de soi-même, car il y a le moi de la veille, de la conscience et celui qui met ce dernier entre parenthèses en agissant de manière plus authentique, plus indestructible même. Car chez Benjamin ainsi que chez Proust, la perception est une sorte de plaque sensible qui a besoin d'une luminosité spécifique pour pouvoir agir et générer des images-traces.

On peut donc dire que la perception a, de la même façon que l'appareil photographique, un double aspect, il y a la perception en état de veille, de la conscience, comme c'est le cas de la photographie des journaux et, d'autre part, la perception de la mémoire involontaire où il y a une mise à part du sujet conscient, une distanciation où se met en place un mécanisme automatique et objectif. Chez Benjamin, dans son texte Chronique berlinoise, la photographie est à nouveau mise en relation avec l'allumette qui, avec un seul geste, prend feu. C'est en définitive la réponse motrice automatique que transmettent les nerf dans l'innervation.

Il en va de même avec Proust chez qui cet automatisme de la perception est identifié à la prise de vue du photographe dans ce célèbre paragraphe de la Recherche:

J'étais là, ou plutôt je n'étais pas encore là puisqu'elle ne le savait pas. [...] De moi par ce privilège qui ne dure pas et où nous avons, pendant le court instant du retour, la faculté d'assister brusquement à notre propre absence - il n'y avait là que le témoin, l'observateur, en chapeau et manteau de voyage, l'étranger qui n'est pas de la maison, le photographe qui vient prendre un cliché des lieux qu'on ne reverra 
plus. Ce qui, mécaniquement, ce fit en ce moment dans mes yeux quand j'aperçus

ma grand-mère, ce fut bien une photographie ${ }^{24}$. intérêt veut se concentrer sur le fait que l'appareil photographique ne peut pas se réduire à ses différents usages qui en effet sont multiples. Ce qu'il faut plutôt mettre en relief c'est son fonctionnement qui fait de l'image photographique une image complètement différente des autres. La photographie est une image-technique, et en tant que telle, elle nous montre un autre aspect du réel qui ne dépend pas nécessairement des capacités humaines. Son automatisme met l'homme et ces capacités entre parenthèses, mais en même temps elle est la maîtresse de l'homme moderne car elle le soumet à un entraînement perceptif nécessaire pour habiter les grandes villes au milieu de la foule.

\section{BIBLIOGRAPHIE}

Baudelaire Charles, « Salon de 1859 » in Euvres complètes, Bruges, Bibliothèque de la Pléiade, 1961. Benjamin Walter, «Sur le théâtre prolétarien » in Profession : Révolutionnaire, Lacis Asja, Grenoble, PUG, 1989.

Benjamin Walter, «L'œuvre d'art à l'époque de sa reproductibilité technique », in Écrits français, Paris, Gallimard, [1936], 1991.

Benjamin Walter, «L'œuvre d'art à l'ère de sa reproductibilité technique », in đEuvres III, traduction de Maurice Gandillac, Rainer Rochlitz et Pierre Rusch, Paris, Gallimard, [1 ${ }^{\text {re }}$ version 1935], 2000.

Benjamin Walter, «Sur quelques thèmes baudelairiens », in Charles Baudelaire, Paris, Payot, 2002. Brassaï, Marcel Proust sous l'emprise de la photographie, Paris, Gallimard, 1997.

Déotte Jean-Louis, « Panofsky : Appareil et prothèse », L'époque des appareils, Paris, Lignes, 2004. 
Fiedler Konrad, Sur l'origine de l'activité artistique, traduction d'Ilena Parvu, Inès Rotermund, Sarah Schmidt, Werner Uwer, Sasha Zilberfarb sous la direction de Danièle Cohn, Paris, Éditions Rue d'Ulm, 2008.

Freud Sigmund, Au-delà du principe du plaisir, traduction de Janine Altounian, André Bourguignon, Pierre Cotet, Alain Rauzy, Paris, PUF, 2010.

Kracauer Siegfried, «La photographie », Théorie du film. La rédemption de la réalité matérielle, Paris, Flammarion, traduction de Daniel Blanchard et Claude Orsoni, 2010.

Laplanche Jean et Pontalis Jean-Bertrand, Vocabulaire de la psychanalyse, Paris, PUF, 1973.

Leroi-Gourhan André, Le geste et la parole. La mémoire et les rythmes, Paris, Albin Michel, 1965.

Morat Jean-Pierre, Traité de physiologie. Fonctions d'innervation, Paris, Masson et Cie éditeurs, 1902.

Proust Marcel, « Le côté de Guermantes », À la recherche du temps perdu, Paris, Robert Laffont, 1987.

\section{NOTES}

1. Siegfried Kracauer, "La photographie ", Théorie du film. La rédemption de la réalité matérielle, Paris, Flammarion, traduction de Daniel Blanchard et Claude Orsoni, 2010, p. 36.

2. Charles Baudelaire, "Salon de 1859 ", in CEuvres complètes, Bruges, Bibliothèque de la Pléiade, 1961, p. 1035.

3. André Leroi-Gourhan, Le geste et la parole. La mémoire et les rythmes, Paris, Albin Michel, 1965, p. 33-34.

4. Ibid., p. 42.

5. Jean-Louis Déotte, « Panofsky : Appareil et prothèse », L'époque des appareils, Paris, Lignes, 2004, p. $167-181$.

6. André Leroi-Gourhan, op. cit., p. 48.

7. Jean-Pierre Morat, Traité de physiologie. Fonctions d'innervation, Paris, Masson et Cie éditeurs, 1902, p. V.

8. Ibid., p. V-VI.

9. Jean Laplanche et Jean-Bertrand Pontalis, Vocabulaire de la psychanalyse, Paris, PUF, 1973, p. 201-202.

10. Walter Benjamin, «Sur le théâtre prolétarien" in Profession: Révolutionnaire, Lacis Asja, Grenoble, PUG, 1989, p. 54-55.

11. En consultant les traductions des Schriften über Kunst de Fiedler, en français Essais sur l'art, et Escritos sobre el arte en espagnol, on se rend compte que le mot « innervation » n'apparaît pas dans le texte. La chercheuse allemande de l'Université de Kontanz Anna Grebe, que je tiens à remercier, a fait la révision de la version allemande du texte en aboutissant au même résultat.

12. Konrad Fiedler, Sur l'origine de l'activité artistique, traduction d'Ilena Parvu, Inès Rotermund, Sarah Schmidt, Werner Uwer, Sasha Zilberfarb sous la direction de Danièle Cohn, Paris, Éditions Rue d'Ulm, 2008, p. 86. Il faut noter que ce texte fait partie des Essais sur l'art.

13. Walter Benjamin, "L'œuvre d'art à l'ère de sa reproductibilité technique », in Euvres III, traduction de Maurice Gandillac, Rainer Rochlitz et Pierre Rusch, Paris, Gallimard, [1 ${ }^{\text {re }}$ version 1935], 2000, p. 81.

14. Ibid., p. 88.

15. Ibid., p. 102.

16. Walter Benjamin, «L'œuvre d'art à l'époque de sa reproductibilité technique ", in Écrits franç ais, Paris, Gallimard, [1936], 1991, p. 226. 
17. Ibid., p. 189.

18. Sigmund Freud, Au-delà du principe du plaisir, traduction de Janine Altounian, André Bourguignon, Pierre Cotet, Alain Rauzy, Paris, PUF, 2010, p. 26.

19. Ibid., p. 24.

20. Walter Benjamin, «Sur quelques thèmes baudelairiens ", in Charles Baudelaire, Paris, Payot, 2002, p. 180.

21. Ibid., p. 179.

22. Loc. cit.

23. Brassaï, Marcel Proust sous l'emprise de la photographie, Paris, Gallimard, 1997, p. 20.

24. Marcel Proust, «Le côté de Guermantes ", À la recherche du temps perdu, Paris, Robert Laffont, 1987, p. 130.

\section{RÉSUMÉS}

La photographie a eu un rôle fondamental en Amérique latine à cause d'une certaine contemporanéité entre l'appareil et la construction des premières nations américaines. Le rôle de la photographie n'est donc pas accessoire et il constitue plutôt une véritable mise en forme de la perception et un outil de construction de la mémoire en Amérique. Parce que le concept d'outil en tant qu'externalisation des capacités humaines nous semble insatisfaisant pour parler de l'appareil photographique et de son rôle, il nous semble nécessaire de recourir au concept d'innervation technique pour rendre compte de la nouvelle perception qui naît dans le Nouveau Monde.

\section{INDEX}

Mots-clés : Amérique latine, photographie, outil-innervation, perception-mémoire

\section{AUTEUR}

\section{NATALIA CALDERÓN}

Doctorante en philosophie, université Paris 8, martinez.talanatalia@gmail.com 